\title{
FIXED PRECISION OPTIMAL ALLOCATION IN TWO-STAGE SAMPLING
}

Abstract. Two-stage sampling schemes arise in survey sampling, especially in situations when the complete update of the frame is difficult. In this paper we solve the problem of fixed precision optimal allocation in two special two-stage sampling schemes. The solution is based on reducing the original question to an eigenvalue problem and then using the PerronFrobenius theorem.

1. Introduction. We consider a finite population consisting of some number of subpopulations. Subpopulations themselves are split into clusters. We want to estimate total values of a variable in the subpopulations. Assume that the (expected) length of the whole sample is fixed. Our aim is to allocate the sample in subpopulations and clusters in such a way that relative errors of the estimates in all subpopulations are the same and as low as possible. Such a problem falls in the following general scheme. Let $z_{i}$ be a vector describing the allocation inside the $i$ th subpopulation and $V_{i}\left(z_{i}\right)$ be the respective relative error. We want to minimize the common value, say $F=$ $V_{i}\left(z_{i}\right)$ (for all $i$ 's), under the constraints $\sum_{i} g_{i}\left(z_{i}\right)=c$, where the $g_{i}$ 's are given functions and $c$ is a constant. Such a problem can be solved in two steps: 1) For each $i$ separately, minimize $V_{i}\left(z_{i}\right)$ under the constraints $g_{i}\left(z_{i}\right)=$ $c_{i}$. Let $V_{i}^{*}\left(c_{i}\right)$ be the solution. 2) Minimize $F=V_{i}^{*}\left(c_{i}\right)$ under the constraints $\sum_{i} c_{i}=c$. Section 2 describes the first phase of the solution while the second phase is examined in Section 3. In Section 4 we present a numerical example. It is related to a survey of small enterprises, led regularly in Poland by Główny Urząd Statystyczny (Central Statistical Office). Indeed, our work was motivated by the need to improve the sampling strategy in this survey.

2000 Mathematics Subject Classification: Primary 62D05.

Key words and phrases: survey sampling, estimator of total variance, subpopulation, minimization with constraints. 
2. Optimal allocation in two-stage sampling. In this section we restrict our considerations to a fixed, finite subpopulation $U=\{1, \ldots, N\}$ partitioned into clusters: $U=U_{1} \cup \ldots \cup U_{M}$. The objective of a survey is the total value $t$ of a variable $\mathcal{Y}$, i.e. $t=\sum_{u \in U} y_{u}$, where $y_{u}$ denotes the value of $\mathcal{Y}$ for unit $u \in U$. Suppose we are to use a two-stage sampling scheme. At the first stage we choose a random sample $V=\left\{U_{1}, \ldots, U_{M}\right\}$ from the population of clusters. The clusters are therefore primary sampling units (PSUs). At the second stage we draw random samples from the previously chosen clusters. Secondary sampling units (SSUs) are elements of $U$.

Throughout this paper, simple random sampling (SRS) means sampling without replacement, with equal probabilities. We consider the following two sampling schemes:

1. stratified SRS (SSRS) at the first stage followed by SRS at the second stage;

2. SRS at the first stage followed by SSRS at the second stage.

In both cases, we seek an optimal allocation subject to constraints on the (expected) size of the final sample and (expected) size of the primary sample. The latter can be expressed either in terms of the number of PSUs or the number of SSUs.

2.1. SSRS-SRS scheme. The population of PSUs is partitioned into strata: $V=W_{1} \cup \ldots \cup W_{H}$. In what follows, the subscript " $h, j, u$ " will refer to the $u$ th SSU in the $j$ th PSU in the $h$ th stratum (we just rearrange objects to make notation readable). Let $\#\left(W_{h}\right)=M_{h}$ be the number of PSUs in the $h$ th stratum and let $m_{h}$ be the number of PSUs sampled from this stratum $(h=1, \ldots, H)$. Write $U_{h, j}$ for the $j$ th PSU in the $h$ th stratum and let $\#\left(U_{h, j}\right)=N_{h, j}\left(j=1, \ldots, M_{h}\right)$. If a PSU $U_{h, j}$ belongs to the primary sample, then we draw a sample of $n_{h, j}$ SSUs from it at the second stage. Write also $N_{h}=\sum_{j \in W_{h}} N_{h, j}$.

Let $\hat{t}$ be the usual $\pi$-estimator (Horvitz-Thompson estimator) of the total $t$. According to the well-known general formula [cf. Särndal, Svensson and Wretman (1992), Ch. 2], the variance of this estimator is given by

$$
\begin{aligned}
D^{2}(\widehat{t})= & \sum_{h=1}^{H}\left(\frac{1}{m_{h}}-\frac{1}{M_{h}}\right) M_{h}^{2} D_{h}^{2} \\
& +\sum_{h=1}^{H} \frac{M_{h}}{m_{h}} \sum_{j=1}^{M_{h}}\left(\frac{1}{n_{h, j}}-\frac{1}{N_{h, j}}\right) N_{h, j}^{2} S_{h, j}^{2},
\end{aligned}
$$

where $t_{h, j}=\sum_{u \in U_{h, j}} y_{h, j, u}, \bar{y}_{h, j}=t_{h, j} / N_{h, j}$ and $\bar{t}_{h}=\sum_{j=1}^{M_{h}} t_{h, j} / M_{h}$, 


$$
S_{h, j}^{2}=\frac{1}{N_{h, j}-1} \sum_{u \in U_{h, j}}\left(y_{h, j, u}-\bar{y}_{h, j}\right)^{2}, \quad D_{h}^{2}=\frac{1}{M_{h}-1} \sum_{j=1}^{M_{h}}\left(t_{h, j}-\bar{t}_{h}\right)^{2} .
$$

The problem is to minimize $D^{2}(\widehat{t})$ as a function of the variables $m_{h}$ and $n_{h, j}$, given that the expected number of SSUs in the primary sample is equal to $n_{I}$ and the expected number of SSUs in the final sample is equal to $n$. Therefore, the constraints are the following:

$$
\sum_{h=1}^{H} \frac{m_{h}}{M_{h}} \sum_{j=1}^{M_{h}} N_{h, j}=n_{I}, \quad \sum_{h=1}^{H} \frac{m_{h}}{M_{h}} \sum_{j=1}^{M_{h}} n_{h, j}=n .
$$

Let $\mathbf{P 1}$ denote the problem described above: minimize (1) subject to (2). Using the standard method of Lagrange multipliers, we easily obtain the following result.

Proposition 1. Provided that $\gamma_{h}=M_{h} D_{h}^{2}-\sum_{j=1}^{M_{h}} N_{h, j} S_{h, j}^{2}>0$ for all $h$, the solution of $\mathbf{P 1}$ has the following form: the optimal allocation is

$$
m_{h}=\frac{M_{h}}{N_{h}} \cdot \frac{\sqrt{N_{h} \gamma_{h}}}{\sum_{k=1}^{H} \sqrt{N_{k} \gamma_{k}}} n_{I},
$$

and

$$
n_{h, j}=n \frac{M_{h}}{m_{h}} \cdot \frac{N_{h, j} S_{h, j}}{\sum_{k=1}^{H} \sum_{l=1}^{M_{k}} N_{k, l} S_{k, l}} .
$$

The minimum value of the variance (1) is

$$
D_{\mathrm{opt}}^{2}(\widehat{t})=\frac{1}{n_{I}}\left(\sum_{h=1}^{H} \sqrt{N_{h} \gamma_{h}}\right)^{2}+\frac{1}{n}\left(\sum_{h=1}^{H} \sum_{j=1}^{M_{h}} N_{h, j} S_{h, j}\right)^{2}-\sum_{h=1}^{H} M_{h} D_{h}^{2} .
$$

A slightly different problem is obtained if we replace the first of the constraints in (2) by a constraint on the expected size of the primary sample expressed in terms of PSUs. Put differently, instead of (2), we consider the following system of constraints:

$$
\sum_{h=1}^{H} m_{h}=m, \quad \sum_{h=1}^{H} \frac{m_{h}}{M_{h}} \sum_{j=1}^{M_{h}} n_{h, j}=n .
$$

Let $\mathbf{P 2}$ denote the problem: minimize (1) subject to (3).

Proposition 2. Provided that $\gamma_{h}>0$ for all $h$, the solution of $\mathbf{P 2}$ has the following form: the optimal allocation of PSUs is

$$
m_{h}=\frac{\sqrt{M_{h} \gamma_{h}}}{\sum_{k=1}^{H} \sqrt{M_{k} \gamma_{k}}} m .
$$


The formula for optimal $n_{h, j}$ is the same as in Proposition 1 . The minimum value of the variance (1) is

$$
D_{\mathrm{opt}}^{2}(\widehat{t})=\frac{1}{m}\left(\sum_{h=1}^{H} \sqrt{M_{h} \gamma_{h}}\right)^{2}+\frac{1}{n}\left(\sum_{h=1}^{H} \sum_{j=1}^{M_{h}} N_{h, j} S_{h, j}\right)^{2}-\sum_{h=1}^{H} M_{h} D_{h}^{2} .
$$

2.2. $S R S-S S R S$ scheme. Now assume that at the first stage PSUs are sampled according to the SRS scheme. Let $M$ be the number of PSUs in the population $V$ and assume the number of PSUs in the primary sample is $m$. Each of the PSUs (clusters) is partitioned into $H$ strata. Let $N_{j, h}$ stand for the number of SSUs in the $h$ th stratum of the $j$ th cluster $\left(h=1, \ldots, H_{j}\right.$, $j=1, \ldots, M$; note that we need a different labeling of units than in the previous subsection). We apply stratified SRS at the second stage, sampling $n_{j, h}$ units from the $h$ th stratum of the $j$ th cluster. The general formula for the variance of the $\pi$-estimator $\widehat{t}$ of the total $t$ now assumes the following form:

$$
D^{2}(\widehat{t})=\left(\frac{1}{m}-\frac{1}{M}\right) M^{2} D^{2}+\frac{M}{m} \sum_{j=1}^{M} \sum_{h=1}^{H_{j}}\left(\frac{1}{n_{j, h}}-\frac{1}{N_{j, h}}\right) N_{j, h}^{2} S_{j, h}^{2},
$$

where $S_{j, h}^{2}$ is the variance of the variable $\mathcal{Y}$ in the $h$ th stratum of the $j$ th PSU and

$$
D^{2}=\frac{1}{M-1} \sum_{j=1}^{M}\left(t_{j}-\bar{t}\right)^{2}
$$

denotes the variance of totals of $\mathcal{Y}$ in PSUs (it is a variable defined in the population $V$ of PSUs). Consider the following constraint on the expected sample size:

$$
\frac{m}{M} \sum_{j=1}^{M} \sum_{h=1}^{H_{j}} n_{j, h}=n .
$$

Denote by P3 the problem of minimizing (4) under constraint (5).

Proposition 3. The allocation problem P3 has the Neyman-type solution

$$
n_{j, h}=n \frac{M}{m} \cdot \frac{N_{j, h} S_{j, h}}{\sum_{l=1}^{M} \sum_{k=1}^{H_{l}} N_{l, k} S_{l, k}} .
$$

The optimal variance is

$$
D_{\text {opt }}^{2}(\widehat{t})=\frac{1}{m} M \gamma+\frac{1}{n}\left(\sum_{j=1}^{M} \sum_{h=1}^{H_{j}} N_{j, h} S_{j, h}\right)^{2}-M D^{2},
$$


where

$$
\gamma=M D^{2}-\sum_{j=1}^{M} \sum_{h=1}^{H_{j}} N_{j, h} S_{j, h}^{2}
$$

If instead of fixing the sample size of PSUs, we fix the expected number of SSUs in the primary sample, i.e.

$$
\frac{m}{M} N=n_{I}
$$

then the formulas for optimal allocation take slightly modified forms. Denote by $\mathbf{P} 4$ the problem of minimizing (4) under constraints (5) and (6), given $n_{I}$ and $n$.

Proposition 4. The allocation problem $\mathbf{P} 4$ has the following solution:

$$
n_{j, h}=n \frac{n_{I}}{N} \cdot \frac{N_{j, h} S_{j, h}}{\sum_{l=1}^{M} \sum_{k=1}^{H_{l}} N_{l, k} S_{l, k}} .
$$

The optimal variance is

$$
D_{\text {opt }}^{2}(\widehat{t})=\frac{1}{n_{I}} N \gamma+\frac{1}{n}\left(\sum_{j=1}^{M} \sum_{h=1}^{H_{j}} N_{j, h} S_{j, h}\right)^{2}-M D^{2} .
$$

Let us sum up the considerations of this section. Observe that the formulas for the optimal variance for problems P1-P4 (see Propositions 1-4) are of the same shape. In what follows we will be interested in the precision of the estimator $\widehat{t}$, defined as $2 \sqrt{D^{2}(\widehat{t})} / t$. Of course, the optimal precision corresponds to the minimal variance. We can express the squared relative error in the following form:

$$
D_{\mathrm{opt}}^{2}(\widehat{t}) / t^{2}=V(x, y)=\frac{A}{x}+\frac{B}{y}-C,
$$

where $x$ and $y$ are allocation parameters $\left(x=n_{I}\right.$ and $y=n$ for $\mathbf{P 1}, \mathbf{P} 4$ and $x=m$ and $y=n$ for $\mathbf{P 2}$, P3) while $A, B$ and $C$ are population parameters.

3. Equating the precision in subpopulations. Now consider a population $U$ partitioned into subpopulations (domains) $D_{i}, i=1, \ldots, L$. We turn to the second stage of our main problem, as described in the introduction. This means that we seek an allocation of the sample into subpopulations which gives equal and optimal precisions. Now, we apply formula (7) separately for each of the domains, arriving at

$$
D_{\mathrm{opt}}^{2}\left(\widehat{t}_{i}\right) / t_{i}^{2}=V_{i}\left(x_{i}, y_{i}\right)=\frac{A_{i}}{x_{i}}+\frac{B_{i}}{y_{i}}-C_{i},
$$

where the additional subscript $i$ indicates the subpopulation $D_{i}$. In particular, $t_{i}$ is the total value of $\mathcal{Y}$ in the domain $D_{i}$ and $\widehat{t}_{i}$ is its estimator. Let 
us recall that $x_{i}$ and $y_{i}$ are allocation parameters, while $A_{i}, B_{i}$ and $C_{i}$ are now subpopulation parameters.

3.1. Main result. Our main problem is to find the smallest common value $F$ of the squared relative error $V_{i}\left(x_{i}, y_{i}\right)$ in the domains $D_{i}, i=1, \ldots, L$. The solution is based on the following theorem.

Theorem 1. Let $x, y$ and $A_{i}, B_{i}, C_{i}, i=1, \ldots, L$, be given positive numbers. Assume that $A_{i} / x+B_{i} / y-C_{i}>0$ for all $i$. Put $a_{i}=\sqrt{A_{i}}$ and $b_{i}=\sqrt{B_{i}}$. The smallest $F>0$ such that

$$
\frac{A_{i}}{x_{i}}+\frac{B_{i}}{y_{i}}-C_{i}=F \quad(\forall i=1, \ldots, L),
$$

under the constraints $x_{i}, y_{i}>0, i=1, \ldots, L$, and

$$
\sum_{i=1}^{L} x_{i}=x, \quad \sum_{i=1}^{L} y_{i}=y,
$$

is the largest eigenvalue of the matrix

$$
\frac{1}{x} \mathbf{a a}^{T}+\frac{1}{y} \mathbf{b b}^{T}-\operatorname{diag}(\mathbf{C}),
$$

where $\mathbf{a}=\left(a_{1}, \ldots, a_{L}\right)^{T}, \mathbf{b}=\left(b_{1}, \ldots, b_{L}\right)^{T}, \mathbf{C}=\left(C_{1}, \ldots, C_{L}\right)^{T}$. This eigenvalue is simple and positive. A corresponding eigenvector $\mathbf{v}=\left(v_{1}, \ldots, v_{l}\right)^{T}$ has all components positive. The values of $x_{i}$ 's and $y_{i}$ 's corresponding to the minimum $F$ are given by

$$
x_{i}=\frac{a_{i} v_{i}}{\sum_{r=1}^{L} a_{r} v_{r}} x, \quad y_{i}=\frac{b_{i} v_{i}}{\sum_{r=1}^{L} b_{r} v_{r}} y .
$$

Proof. Consider the function $f:(0, \infty)^{2 L+1} \rightarrow \mathbb{R}$ defined by

$$
f\left(x_{1}, \ldots, x_{L}, y_{1}, \ldots, y_{L}, F\right)=F .
$$

We seek the minimum of $f$ on the set

$$
\mathcal{R}=\left\{\left(x_{1}, \ldots, x_{L}, y_{1}, \ldots, y_{L}, F\right) \in(0, \infty)^{2 L+1}:(8) \text { and (9) hold }\right\} .
$$

It is clear that $f$ is bounded from below on this set, because $A_{i} / x_{i} \geq$ $A_{i} / x$ and $B_{i} / y_{i} \geq B_{i} / y$. Let $f^{*}$ be the infimum of $f$ over $\mathcal{R}$. Notice that $\left\{\left(x_{1}, \ldots, x_{L}, y_{1}, \ldots, y_{L}, F\right) \in \mathcal{R}: F \leq f^{*}+1\right\}$ is a compact set. Indeed, it is a (relatively) closed and bounded subset of $(0, \infty)^{2 L+1}$. Moreover, this set is bounded away from the boundary of $(0, \infty)^{2 L+1}$, because $x_{i} \rightarrow 0$ or $y_{i} \rightarrow 0$ together with (8) implies $F \rightarrow \infty$. Therefore, there exists a point $\left(x_{1}^{*}, \ldots, x_{L}^{*}, y_{1}^{*}, \ldots, y_{L}^{*}, F^{*}\right) \in \mathcal{R}$ such that $F^{*}=f^{*}$. This is a stationary point of $f$, under constraints (8) and (9). Using the method of Lagrange multipliers, we will find it and then show it is unique. 
Consider the Lagrange function

$$
F+\sum_{i=1}^{L} \lambda_{i}\left(\frac{A_{i}}{x_{i}}+\frac{B_{i}}{y_{i}}-C_{i}-F\right)+\alpha\left(\sum_{i=1}^{L} x_{i}-x\right)+\beta\left(\sum_{i=1}^{L} y_{i}-y\right),
$$

where $\alpha, \beta$ and $\lambda_{i}$ are the Lagrange multipliers. Differentiating (10) with respect to $x_{i}$ and $y_{i}$, we infer that $\alpha\left(x_{i}^{*}\right)^{2}=\lambda_{i} A_{i}$ and similarly $\beta\left(y_{i}^{*}\right)^{2}=$ $\lambda_{i} B_{i}$. Hence all the Lagrange multipliers $\alpha, \beta$ and $\lambda_{i}$ have the same sign. Without loss of generality we can assume that they are all positive. Let us write $v_{i}=\sqrt{\lambda_{i}}$ and recall that $A_{i}=a_{i}^{2}, B_{i}=b_{i}^{2}$. We arrive at

$$
x_{i}^{*}=\frac{a_{i} v_{i}}{\sqrt{\alpha}}, \quad y_{i}^{*}=\frac{b_{i} v_{i}}{\sqrt{\beta}} .
$$

Use constraints (9) to get $\sqrt{\alpha}=\sum_{r=1}^{L} a_{r} v_{r} / x$ and $\sqrt{\beta}=\sum_{r=1}^{L} b_{r} v_{r} / y$. Now, plug in the expressions for $x_{i}^{*}$ and $y_{i}^{*}$ into (8). We obtain

$$
\frac{a_{i}}{x v_{i}} \sum_{r=1}^{L} a_{r} v_{r}+\frac{b_{i}}{y v_{i}} \sum_{r=1}^{L} b_{r} v_{r}-C_{i}=F^{*}
$$

or, equivalently,

$$
\sum_{r=1}^{L}\left(\frac{a_{i} a_{r}}{x}+\frac{b_{i} b_{r}}{y}\right) v_{r}-C_{i} v_{i}=F^{*} v_{i} .
$$

In matrix notation, this is just

$$
\left(\frac{1}{x} \mathbf{a a}^{T}+\frac{1}{y} \mathbf{b b}^{T}-\operatorname{diag}(\mathbf{C})\right) \mathbf{v}=F^{*} \mathbf{v},
$$

where $\mathbf{v}=\left(v_{1}, \ldots, v_{L}\right)^{T}$. Under our assumptions, all elements of the symmetric matrix $\mathbf{M}=\mathbf{a a}^{T} / x+\mathbf{b b}^{T} / y-\operatorname{diag}(\mathbf{C})$ are positive. By the PerronFrobenius theorem, the largest eigenvalue of $\mathbf{M}$ is simple and it is the only eigenvalue with a positive corresponding eigenvector [cf. Kato (1981), Th. 7.3 in Ch. 1]. Hence it must be $F^{*}$ and the corresponding eigenvector is $\mathbf{v}$. The conclusions of the theorem follow.

3.2. Corollaries. Now, let us specify the conclusions of Theorem 1 to the four problems considered in Section 2. We only have to express the parameters $a_{i}, b_{i}$ and $C_{i}$ using the formulas for the optimum variance for P1-P4, respectively. The formulas are given in Propositions 1-4. Note that now the additional subscript $i$, referring to the subpopulation, has to appear in each of these formulas $(i=1, \ldots, L)$.

For $\mathbf{P} 1$ we have

$$
a_{i}=\sum_{h=1}^{H_{i}} \sqrt{N_{i, h} \gamma_{i, h}} / t_{i}, \quad b_{i}=\sum_{h=1}^{H_{i}} \sum_{j=1}^{M_{i, h}} N_{i, h, j} S_{i, h, j} / t_{i},
$$




$$
C_{i}=\sum_{h=1}^{H_{i}} M_{i, h} D_{i, h}^{2} / t_{i}^{2}, \quad x=n_{I}, \quad y=n .
$$

Consequently, the solution of our fixed precision optimal allocation problem is given by

$$
\begin{aligned}
m_{i, h} & =x_{i} \frac{M_{i, h}}{N_{i, h}} \cdot \frac{\sqrt{N_{i, h} \gamma_{i, h}}}{\sum_{k=1}^{H_{i}} \sqrt{N_{i, k} \gamma_{i, k}}}, \\
n_{i, h, j} & =y_{i} \frac{M_{i, h}}{m_{i, h}} \cdot \frac{N_{i, h, j} S_{i, h, j}}{\sum_{k=1}^{H_{i}} \sum_{l=1}^{M_{i, k}} N_{i, k, l} S_{i, k, l}} .
\end{aligned}
$$

For $\mathbf{P} 2$ we have $b_{i}, C_{i}$ and $y$ the same as above,

$$
a_{i}=\sum_{h=1}^{H_{i}} \sqrt{M_{i, h} \gamma_{i, h}} / t_{i}, \quad x=m .
$$

The solution of the fixed precision optimal allocation problem is given by

$$
m_{i, h}=x_{i} \frac{\sqrt{M_{i, h} \gamma_{i, h}}}{\sum_{k=1}^{H_{i}} \sqrt{M_{i, k} \gamma_{i, k}}}
$$

and $n_{i, h, j}$ as in (11).

For P3 we have

$$
\begin{gathered}
a_{i}=\sqrt{M_{i} \gamma_{i}} / t_{i}, \quad b_{i}=\sum_{j=1}^{M_{i}} \sum_{h=1}^{H_{i, j}} N_{i, j, h} S_{i, j, h} / t_{i}, \\
C_{i}=M_{i} D_{i}^{2} / t_{i}^{2}, \quad x=m, \quad y=n .
\end{gathered}
$$

The solution of the fixed precision optimal allocation problem is given by

$$
m_{i}=x_{i}, \quad n_{i, j, h}=y_{i} \frac{M_{i}}{m_{i}} \cdot \frac{N_{i, j, h} S_{i, j, h}}{\sum_{l=1}^{M_{i}} \sum_{k=1}^{H_{i, l}} N_{i, l, k} S_{i, l, k}} .
$$

For $\mathbf{P} \mathbf{4}$ we have $b_{i}, C_{i}$ and $y$ as above,

$$
a_{i}=\sqrt{N_{i} \gamma_{i}}, \quad x=n_{I}
$$

The solution of the fixed precision optimal allocation problem is given by

$$
m_{i}=x_{i} \frac{M_{i}}{N_{i}}
$$

and $n_{i, j, h}$ as in (12).

4. Numerical example. We consider a population of small (up to 5 employees) enterprises in a southern part of Poland of size 79578, divided into 4 subpopulations, say A, B, C, and D, of respective sizes 15144, 11582, 26960, 25892. Each of the subpopulations is clustered into regions. There are 170 regions in A, 128 in B, 306 in C and 326 in D, thus the total number 
of regions equals 930 . In each of the subpopulations $\mathrm{A}, \mathrm{B}, \mathrm{C}$ and $\mathrm{D}$, the regions are stratified into $8,7,8$ and 8 strata, respectively. The variable of interest is the total employment in subpopulations. We seek a fixed precision optimal allocation of the sample using the double stage SSRS-SRS scheme where PSUs are regions and SSUs are enterprises. We impose the following constraints: the total size of the primary unit sample is 10000 SSUs and the total size of the final sample equals 2000 SSUs.

Thus we are in the framework of the problem $\mathbf{P} \mathbf{1}$ discussed above with $n_{I}=10000, n=2000$ and $L=4$. First it was checked that all $\gamma_{h}$ 's are positive. Using the definitions of the vectors (4-variate in our setting) $\mathbf{a}, \mathbf{b}$ and $\mathbf{C}$ it was found that

$$
\begin{aligned}
\mathbf{a} & =(2.75441,2.50996,2.59874,2.57227)^{T}, \\
\mathbf{b} & =(0.62892,0.60228,0.61864,0.60082)^{T}, \\
\mathbf{C} & =(0.00063,0.00059,0.00028,0.00028)^{T} .
\end{aligned}
$$

The eigenvalue problem has the following solutions $\left(\lambda_{i}\right.$ denotes the eigenvalue and $\mathbf{v}_{i}$ a respective eigenvector):

$$
\begin{array}{ll}
\lambda_{1}=0.00304, & \mathbf{v}_{1}=(0.49649,0.46302,0.52303,0.51531)^{T}, \\
\lambda_{2}=0.00028, & \mathbf{v}_{2}=(0.00203,0.00183,-0.70362,0.71057)^{T}, \\
\lambda_{3}=0.00045, & \mathbf{v}_{3}=(0.46661,0.57090,-0.47862,-0.47674)^{T}, \\
\lambda_{4}=0.00061, & \mathbf{v}_{4}=(-0.73197,0.67800,0.04772,0.04759)^{T} .
\end{array}
$$

Now using the solution given in the preceding section we notice that $F^{*}=$ $\lambda_{1}$, consequently the precision of the estimate equals $2 \sqrt{F^{*}}=0.1102$. The allocation of the primary sample was computed according to the first part of the formula (11), in terms of the eigenvector $\mathbf{v}_{1}$ (and suitably rounded). For clarity, we present it in the form of pairs $\left(M_{i, h}, m_{i, h}\right)$ :

- for the subpopulation A:

$$
(14,2),(22,3),(2,1),(29,3),(11,2),(71,13),(3,1),(18,4) \text {, }
$$

- for the subpopulation B:

$$
(14,3),(27,5),(31,6),(3,1),(41,10),(2,1),(10,2),
$$

- for the subpopulation C:

$$
(22,2),(46,5),(3,1),(62,5),(13,1),(121,15),(5,1),(34,3) \text {, }
$$

- for the subpopulation D:

$(25,2),(38,3),(2,1),(70,6),(6,1),(154,18),(6,1),(25,3)$.

Similarly the allocation of the final sample of SSUs was obtained using the second part of (11) (and again rounded). Since this is a lengthy table, 
we present here the pairs $\left(N_{i, h, j}, n_{i, h, j}\right)$ only for stratum number 8 of the subpopulation A:

$$
\begin{aligned}
& (127,21),(126,13),(80,2),(11,17),(107,31),(114,17),(123,18),(87,6), \\
& (112,18),(139,17),(113,12),(127,10), \quad(87,9), \quad(119,13), \quad(139,25), \\
& (119,9),(226,33),(145,17) .
\end{aligned}
$$

\section{References}

C. E. Särndal, B. Swensson and J. Wretman (1992), Model Assisted Survey Sampling, Springer, Berlin.

T. Kato (1981), A Short Introduction to Perturbation Theory for Linear Operators, Springer, New York.

Wojciech Niemiro

Institute of Applied Mathematics

and Mechanics

Warsaw University

Banacha 2

02-097 Warszawa, Poland

E-mail: wniem@mimuw.edu.pl

Central Statistical Office

Al. Niepodległości 208

00-925 Warszawa, Poland
Jacek Wesołowski

Faculty of Mathematics and

Information Science Warsaw University of Technology

Pl. Politechniki 1

00-661 Warszawa, Poland

Central Statistical Office

Al. Niepodległości 208

00-925 Warszawa, Poland

Received on 24.2.2000;

revised version on 29.6.2000 